\title{
Focus on diagnosis, treatment, and problems of Barré-Lièou syndrome: Two case reports
}

\author{
Yusuke Morinaga*, Kouhei Nii, Kimiya Sakamoto, Ritsurou Inoue, Takafumi Mitsutake, \\ Hayatsura Hanada
}

Department of Neurosurgery, Fukuoka University Chikushi Hospital, Chikushino-city, Fukuoka, Japan.

\begin{abstract}
Summary Barré-Lièou syndrome is a manifestation of various autonomic and secondary symptoms, such as muscle stiffness, tinnitus, dizziness, and pain in the head, neck, eyes, throat, ears, chest, and back. While thought to be caused by hyperactivation of the autonomic nervous system due to trauma, there is currently no firmly established etiology. This, and the nonspecific nature of many of its symptoms, presents a challenge both for clinicians, who must provide a correct diagnosis and patients, who are often misdiagnosed or faced with undue scrutiny from insurance companies. Here, we present two cases of BarréLièou syndrome, focusing on the processes leading to diagnosis, treatment, and problems encountered. Case 1 involves a 68-year-old woman whose head computed tomography (CT) scan revealed no abnormalities following a car accident. Approximately 10 months after her initial injury, Barré-Lièou syndrome was suspected because of autonomic symptoms that developed over time. She was prescribed an $\alpha$-blocker, and 9 months later, her symptoms subsided. Case 2 was a 69-year-old woman who presented with bruising to the right chest and right knee after colliding with a car while riding her bicycle. One month later, BarréLièou syndrome was suspected because of her autonomic symptoms. She was prescribed an $\alpha$-blocker, and 17 months later, her symptoms subsided. Because of the characteristic autonomic and secondary symptoms described above and a positive response to $\alpha$-blockers, Barré-Lièou syndrome was suspected in both cases. We believe reporting cases will aid in the understanding of this disease and help patients obtain positive outcomes.
\end{abstract}

Keywords: Autonomic symptoms, Barré-Lièou syndrome, indefinite complaints, traumatic cervical syndrome

\section{Introduction}

Barré-Lièou syndrome (1) is a condition first reported by a French neurologist, Jean Alexandre Barré, in 1926 and Chinese physician, Yong-Cheon Lièou, in 1928. Various autonomic and secondary symptoms, such as muscle stiffness, tinnitus, and dizziness, are observed in addition to pain in the head, neck, eyes, throat, ears, chest, and back. It is thought that hyperactivation of the autonomic nervous system (mainly the sympathetic nerves) is directly or indirectly stimulated by trauma.

\footnotetext{
*Address correspondence to:

Dr. Yusuke Morinaga, Department of Neurosurgery, Fukuoka University Chikushi Hospital, 1-1-1 Zokumyoin, Chikushinocity, Fukuoka Prefecture, 818-8502, Japan.

E-mail: yu_the_morio@yahoo.co.jp
}

Numerous theories exist, such as the cervical sympathetic hyperactivity theory, theory of disorders of vertebral artery circulation, theory of brainstem disorder, and cervical soft tissue hypertonia theory. However, a solid theory has not yet been established $(1,2)$.

In this paper, we present two cases of Barré-Lièou syndrome and report on literature considerations, focusing on the process leading to diagnosis and treatment, and issues that can occur during this process.

\section{Case Report}

\subsection{Cases 1}

In Case 1, a 68-year-old woman, with no history of medical conditions, visited to our hospital after being 

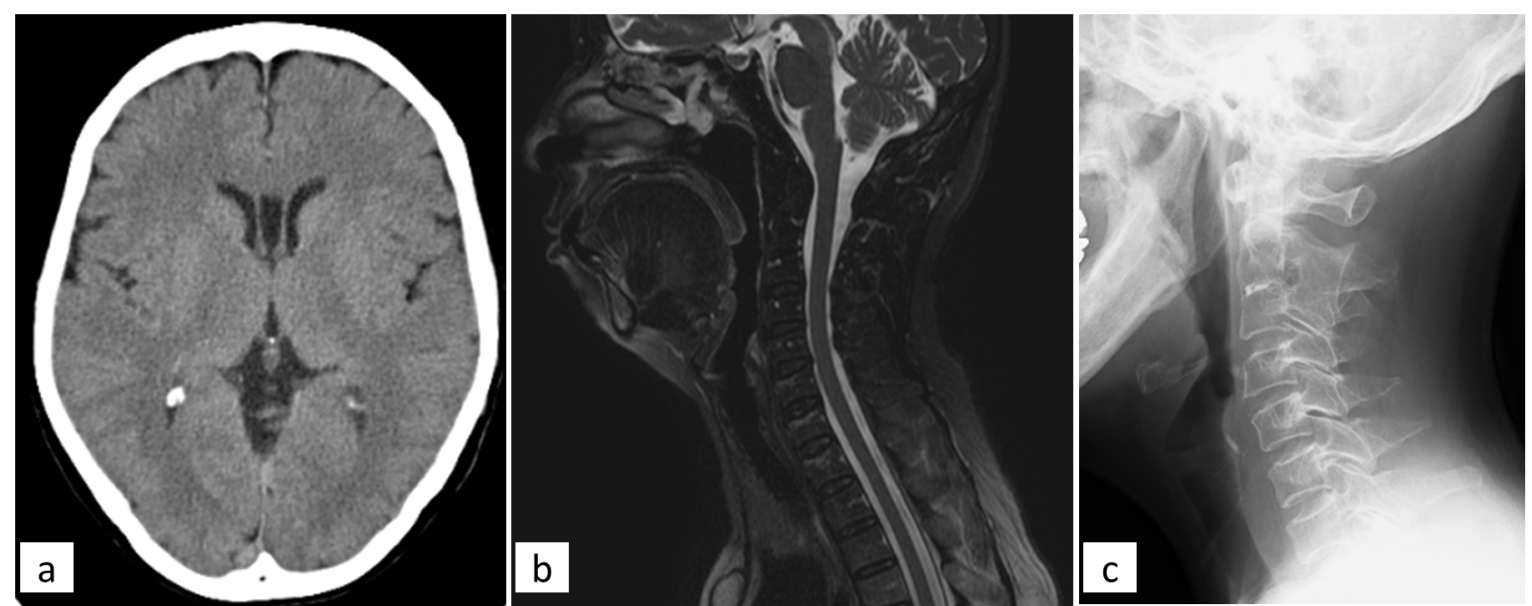

Figure 1. Imaging results for Case Study 1 patient. (a) Head computed tomography (CT) scan. Patient's first visit to our hospital after injury shows no traumatic intracranial hemorrhages or fractures. (b) T2-weighted Magnetic Resonance Image (MRI) after patient presented to our neurosurgery department 10 months after her initial injury. It shows that cervical vertebrae alignment is normal, there is no intramedullary abnormal signal, and C2-3 vertebral-facet joints are fused. The intervertebral discs at $\mathrm{C} 4$ / 5, C5 / 6 mildly bulge, but there is no significant excavation into the dural sac. (c) Cervical spine X-ray, 10 months after injury, shows no fractures or deviations.

injured when her car collided with a tree in an attempt to avoid a bicycle. Head computed tomography (CT) scan showed no fractures and she returned home. Approximately one month after the injury, she presented to the ophthalmology department with blurry vision and discomfort and discharge in both eyes. Cervical spine magnetic resonance imaging (MRI) was performed approximately 2 months after her injury and it showed normal. She was admitted to the department of internal medicine with chest and back pain approximately 3 months after injury. The following month, she began experiencing headaches, posterior head and neck pain, dizziness, pharyngeal and laryngeal discomfort, and pain when swallowing. She also developed insomnia and anxiety and began sleeping all day. Approximately 10 months after injury, she presented to our neurosurgery department for the first time due to her persisting symptoms.

At this first visit, the patient presented fully conscious. Physical findings included a blood pressure of $150 / 92 \mathrm{~mm} \mathrm{Hg}$, and a body temperature of $36.2^{\circ} \mathrm{C}$. A headache was described as a dull, long-lasting pain (more than $72 \mathrm{~h}$ ) with a sense of heaviness centered on the back, head, and neck. The nature of her headache was not pulsatile, strangulating, or shocking. She did not have an orthostatic headache, or nystagmus at rest, or while gazing. She had no apparent symptoms of a neurological deficit, no pathological reflexes, and no tinnitus. She had dizziness without cervical rotation, or induction by posture transformation. Hyperemia and discharge were present in both eyes. Additionally, she had constipation after trauma. A variety of subjective symptoms, such as discomfort in both eyes, chest and back pain, headache, dorsal neck pain, dizziness, pharyngeal and laryngeal discomfort, pain when swallowing, insomnia, and anxiety, were also reported by the patient.

Her blood test revealed no obvious, abnormal findings regarding blood count, biochemistry, or coagulation. A head CT scan at her first visit to our hospital showed no traumatic intracranial hemorrhages or fractures (Figure 1a). We additionally performed a cervical vertebral T2-weighted MRI (Figure 1b). This scan showed normal cervical spine alignment, normal intramedullary signal, and fusion of the vertebral-facet joint of $\mathrm{C} 2-3$. In $\mathrm{C} 4 / 5, \mathrm{C} 5 / 6$ the intervertebral discs mildly bulged, but there were no significant retractions of the dural sac. Additionally, carotid ultrasonography of the vertebral artery indicated no obvious circulatory insufficiency.

Due to the absence of orthostatic headache and the nature of other accompanying symptoms, intracranial hypotension syndrome was not suspected. At the initial visit to our department, Barré-Lièou syndrome was suspected based on the diverse autonomic symptoms and secondary symptoms after trauma. Trazodone $(50 \mathrm{mg} /$ day), an oral sympathetic nervous system and $\alpha$-receptor blocker, was started. Soon after, the majority of her symptoms disappeared, however, occasionally chest, pharyngeal, and laryngeal pain reemerged. Because she did not reach complete remission, and since her systolic blood pressure remained at a safe level, around 120-130 $\mathrm{mm} \mathrm{Hg}$, we continued oral administration of trazodone.

Approximately 13 months after the initial injury, the patient was again injured, this time in a twocar collision, and visited an orthopedist. No obvious fractures or deviations were observed in the cervical spine X-ray image (Figure 1c). However, neck pain, discomfort in the pharynx and larynx, pain when swallowing, and insomnia worsened. We suspected the exacerbation of Barré-Lièou syndrome.

With this second injury triggered, compensation 

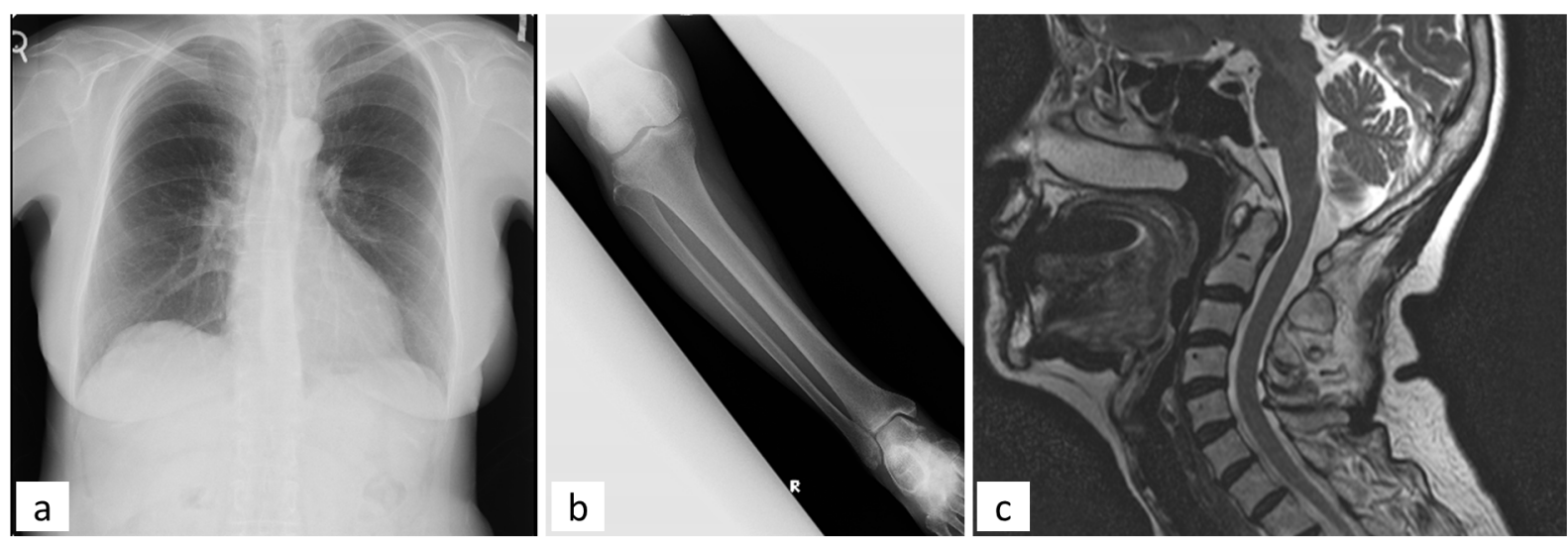

Figure 2. Imaging results for Case Study 2 patient. (a) Chest and (b) right leg X-rays show no fractures or deviations upon initial visit after injury. (c) T2-weighted Magnetic Resonance Image (MRI), taken 1 month after injury, shows normal cervical vertebrae alignment, no intramedullary abnormal signal, and C1-3 vertebral-facet joints are fused. The intervertebral discs at C4 / 5, C5 / 6 mildly bulge, but there is no significant excavation into the dural sac.

from the insurance company, for the initial injury, was canceled. Our department issued a diagnosis document to the insurance company proclaiming, "this patient was diagnosed with, and is being treated for, Barré-Lièou syndrome after her first injury. Her current symptoms are an exacerbation of this disease caused by the second injury." However, the insurance company declined to continue compensation following the second injury. The patient was exhausted from negotiations with the insurance company and was temporarily depressed. She wished to avoid trouble with this company and switched to an individual health insurance plan. She is currently being followed up as an outpatient because, approximately 19 months after her first injury, all her symptoms have disappeared.

\subsection{Case 2}

Case 2 involves a 69-year-old woman with no history of adverse medical conditions. She collided with a car, while riding her bicycle, and presented to our emergency room outpatient facility. Dizziness, neck pain, chest and back pain, pharyngeal and laryngeal discomfort, and pain when swallowing emerged the day following the injury, and she returned home after being diagnosed with right chest and right knee bruising, supported by X-ray results. Then, without improvement of chest pain and back pain, she visited the emergency room in our hospital again. The cause could not be identified with chest and abdominal contrast CT. Four days after the injury, she visited an orthopedist, but no abnormality was pointed out. She had discomfort in and hyperemia of both eyes, and her insomnia and anxiety symptoms appeared to be caused by exacerbation of autonomic symptoms 10 days after injury. Approximately 1 month after the injury, she visited our neurosurgery department hoping to improve her symptoms.

The patient presented fully conscious at her initial visit. Physical findings indicated her blood pressure was $130 / 82 \mathrm{~mm} \mathrm{Hg}$ and her body temperature was $36.1^{\circ} \mathrm{C}$. A headache was described as a dull, long-lasting (more than 72 hours) pain with a sense of heaviness, centered on the back of the head and neck. The nature of her headache was not pulsatile, strangulating, or shocking. She had no orthostatic headache, no nystagmus at rest or while gazing, no apparent neurological deficit symptoms, no pathological reflexes, and no tinnitus. She had dizziness without cervical rotation or induction by posture transformation. Hyperemia of both eyes and eye discharge were found. She also had constipation after trauma.

The patient reported subjective symptoms, including discomfort of both eyes, chest and back pain, headache, dorsal neck pain, dizziness, pharyngeal and laryngeal discomfort, pain when swallowing, insomnia, and anxiety.

The patient's blood test revealed no obvious abnormalities regarding blood count, biochemistry, or coagulation. X-ray of the chest and right lower thigh indicated no apparent fractures or displacements (Figures $2 \mathrm{a}$ and $2 \mathrm{~b}$ ). Cervical vertebral T2-weighted MRI showed normal cervical spine alignment, no abnormal intramedullary signal, and fusion of the vertebral-facet joint of C1-3 (Figure 2c). Finally, carotid ultrasonography indicated no obvious circulatory insufficiency in the vertebral artery.

Due to the absence of orthostatic headache and the nature of other accompanying symptoms, a diagnosis of intracranial hypotension syndrome was excluded. During this visit, Barré-Lièou syndrome was suspected based on her diverse autonomic and secondary symptoms after trauma. An oral drug, which is a sympathetic nervous system blocker, trazodone (50 $\mathrm{mg} /$ day), was started. Following treatment, her blurriness, discharge, and discomfort in both eyes, headache, dorsal neck pain, dizziness, insomnia, anxiety, and constipation disappeared. However, occasionally back 
pain reappeared, therefore, she continued oral trazodone administration.

Six months after the injury, the insurance company became suspicious of her symptoms and, as with our Case 1 patient, proposed canceling compensation. The patient worried about responding to the insurance company and decided to stop oral administration because her symptoms had improved. Her back pain worsened afterwards, and she went to an osteopathic clinic of her own volition, but her symptoms did not improve. After restarting $50 \mathrm{mg} /$ day of trazodone, her symptoms disappeared, and complete remission was obtained approximately 18 months after injury.

Written informed consent was obtained from the patients for the publication of these two case reports and the accompanying images, and the study design was approved by the appropriate ethics review board.

\section{Discussion}

Barré-Lièou syndrome is a refractory disease that exhibits cervical pain and various autonomic and secondary symptoms (insomnia, anxiety, depression, etc.) and is considered a subtype of traumatic cervical syndrome (1). The Quebec classification is wellknown for its scoring of traumatic cervical syndrome, classifying severity between grades 0 - IV (3). We recommend an early exit from bed in cases of grade III or lower without fracture, dislocation, or spinal cord injury, and discontinuation of early treatment within 3 months (2). As a subjective and nonspecific complaint, Barré-Lièou syndrome is liable to be an indefinite complaint as it lacks objective neurological findings and biomarkers. The symptoms are as follows: (1) inner ear symptoms: dizziness, tinnitus, and feelings of obstruction of the ear; (2) eye symptoms: blurred vision, fatigue, discomfort, and sight loss (asthenopia); (3) chest and back symptoms: chest pain, back pain, arrhythmia, and respiratory distress; (4) pharyngeal and laryngeal symptoms: hoarseness, discomfort in the throat, and swallowing difficulty; (5) head and neck symptoms: headache, sense of head weight, and back of neck pain; (6) others: upper limb and whole body fatigue, upper limb numbness, distraction, secondary psychiatric symptoms (anxiety, depression, and the like), and insomnia (4).

It is important to note that intracranial hypotension syndrome (5), which exhibits symptoms such as headache, dizziness, and nausea when standing because of a decrease in cerebrospinal fluid volume, has clinical characteristics similar to those of Barré-Lièou syndrome. In these cases, a cisternography should be performed for accurate diagnoses. There are reports that epidural blood patch therapy for intracranial hypotension syndrome is effective treatment (2).

In general, a clear diagnosis is difficult, but some symptoms that can aid diagnosis exist: (1) symptoms that can be explained as autonomic symptoms (however these are mainly subjective, and objective findings are poor); (2) positive response to sympathetic blockade drugs and sympathetic ganglion blocks (mainly symptomatic improvement may be seen by the stellate ganglion block); (3) abnormal imaging results, such as a fracture or dislocation. (4) circulatory failure of the vertebral artery feeding the brainstem (especially cranial nerves V2, 3 and VIII etc.) where the central autonomic network exists (especially C3, 4 vertebral bodies, intervertebral disc trauma, arthritis, etc.) caused by temporal changes of the cervical spine. If these symptoms exist, the likelihood of Barré-Lièou syndrome will be even higher (4).

In both our cases, carotid artery echoes were negative in the vertebral arterial circulation in the neck, there were no orthostatic headaches, and considering the existence of other accompanying symptoms, intracranial hypotension syndrome was ruled out. Case 1 and Case 2 satisfy all the above criteria (1-4), and a Quebec grade of X Barré-Lièou syndrome was suspected. Furthermore, the fact that trazodone, an oral sympathetic nervous system blocker, was effective supports the cervical sympathetic hyperactivity theory.

Treatment is basically supposed to follow the treatment protocol for cervical sprains, but a stellate ganglion block (one of the cervical sympathetic ganglia) or an $\alpha$-blocker (a sympatholytic drug) is recommended. In addition, various treatments such as neck epidural block, intravenous drip infusion of prostaglandin E1 (PGE 1), acupuncture, and the like are performed, but a clear treatment effect is not obtained and there are many refractory cases (2). The mental symptoms, insomnia, and autonomic symptoms in our patients disappeared after administration of trazodone. Trazodone is currently effective in both cases, and the stellate ganglion block was not enforced, but will be examined in the future if symptoms exacerbate.

Physicians and patients do encounter problems with the diagnosis of Barré-Lièou syndrome. Because there are few image diagnostics or neurological objective findings, symptoms are often misunderstood as fraudulent to receive compensation for a traffic injury (3). In both our cases, the insurance companies proposed stopping compensation. In Case 1, after a second traffic accident, the patient did not receive compensation despite our letters to the appropriate insurance companies. In Case 2, since her insurance company repeatedly proposed cancelling compensation, the patient stopped drug treatment and sought alternative therapy from an osteopathic clinic, but she later resumed oral administration due to exacerbation of back pain.

As in our cases, patients suspected of fraud often experience litigation problems, and for the same reasons as above, the question of whether disability is residual or primary frequently arises. Furthermore, because this syndrome presents with autonomic symptoms, 
psychiatric symptoms, insomnia, and so on, this syndrome is not only treated as an indefinite complaint, but also many physicians who are unfamiliar with this disease do not establish a confirmed diagnosis (5). As mentioned above, its pathophysiology has not been established, so it has not been recognized internationally as an independent syndrome. In the clinical setting, Barré-Lièou syndrome can be diagnosed as a tensiontype headache, cervical arm syndrome, traumatic cervical syndrome, or an autonomic imbalance. These conditions are not fully understood, and in that respect, it may be worth considering this syndrome as well.

In our opinion, irrespective of the disease name, we make our determination based on clinical symptoms, and we suspected Barré-Lièou syndrome in the patients who presented with refractory symptoms in this report. We believe that it is worth trying trazodone for posttraumatic sympathetic hyperactivity. In addition, when empirical treatment is successful, informed consent of patients based on the characteristics and problems of the disease is also important. To solve such problems, it is desirable to disseminate information about the pathology to better recognize and increase understanding of the disease by physicians and society.

In conclusion, we reported two cases of Barré-Lièou syndrome from the viewpoint of diagnosis, treatment, and problems, in the context of current literature. Diagnosis was delayed as both patients complained of psychiatric symptoms and had indefinite complaints in the form of a variety of autonomic symptoms, such as headache, eye pain, vision disorder, otic pain, tinnitus, dizziness, back of head pain, discomfort, constipation, insomnia, and anxiety. Trazodone, which is an $\alpha$-blocker for Barré-Lièou syndrome, was effective in relieving our patients' symptoms. Enhanced recognition and understanding of this disease by physicians and society are desired.

\section{Acknowledgements}

This research did not receive any specific grant from funding agencies in the public, commercial, or not-forprofit sectors. We would like to thank Editage (www. editage.com) for English language editing.

\section{References}

1. Li Y, Peng B. Pathogenesis, diagnosis, and treatment of cervical vertigo. Pain Physician. 2015; 18:E583-E595.

2. Ishikawa S, Katayama D, Takahara H, Kojo S, Moriyama E, Hashimoto H. Epidural blood patch as a successful treatment of Barrè-Lièou syndrome: report of two cases. Masui. 2003; 52:1305-1311.

3. Spitzer WO, Skovron ML, Salmi LR, Cassidy JD, Duranceau J, Suissa S, Zeiss E. Scientific monograph of Quebec task force on whiplash-associated disorder: Redefining "whiplash" and its management. Spine. 1995; 20:1S-73S.

4. Pearce JM. Barré-Liéou "syndrome". J Neurol Neurosurg Psychiatry. 2004; 75:319.

5. Foster CA, Jabbour P. Barré-Liéou syndrome and the problem of the obsolete eponym. J Laryngol Otol. 2007; 121:680-683.

(Received June 19, 2019; Revised August 13, 2019; Accepted August 22, 2019) 